by many African tribes as a source of arrow poison and as a tonicardiac during periods of violent exercise such as iron smelting or prolonged ceremonies.

The highly restricted diversity of plant species carried into Paouan cave, allied to their known properties and modern uses by tropical African tribes, indicate that we may have discovered a place in which magical-ritual practices were carried out around 5,600 years ago. The presence of worked stone and Strophanthus charcoal suggests that poison arrows were manufactured in the cave. Poison-arrow manufacture by many African tribes is carried out with elaborate ritual in secret places, such as caves, rock shelters or forest thickets, from which women are excluded. The choice of Copaifera resin as the preferred torch-making material, and of $P$. tinctorius accords with the view that Paouan cave was used for magical-ritual purposes.

It seems that by 5,600 years ago, some of the tribes of tropical Africa had mastered the art and phytochemistry of poison-arrow manufacture, and that they had developed elaborate rituals around this activity.

Richard Osilsly

Paléogab, 4 rue Niger, Paris, France

Martin Pickford

Geological Survey of Namibia,

POBox 2168, Windhoek, Namibia

Roger Dechamps

RoyalAfrican Museum,

BP 1980, Tervuren, Belgium

Michel Fontugne

Laboratoire Mixte CNRS-CEA,

Centre des Faibles Radio Activités, 91198 Gif-sur-Yvette, France

1. Delorme, G. Spelunca 4, 151-160 (1979).

2. Van Noten, F. (ed.) in The Archaeology of Central Africa 27-40 (Academische, Gruz, 1982).

\section{Parsimony or statistics?}

SIR - In the light of Stewart's Review article $^{1}$ on parsimony, I would like to comment on the use of this technique with molecular sequence data. Phylogenetic inference, no matter which method is applied, hinges on the tenet that the method used takes into account the mode of evolution of the molecule analysed. To infer phylogenies from DNA sequence data, there must be a mathematical description of the evolutionary process - a stochastic model.

Such models have been incorporated into statistical methods of phylogenetic inference, such as maximum likelihood ${ }^{2,3}$ or corrections of pairwise distances ${ }^{4}$. Methods with general models have wide applicability, allowing analysis of sequences that have undergone few or many substitutions, that evolve by a transition bias, whose positions evolve at different rates, or whose base composition is biased. By contrast, parsimony's implicit stochastic model is highly restrictive. For example, it requires that each nucleotide position has a negligible probability of having changed more than once ${ }^{5}$. It generally is not known a priori if evolution generated one's data in accord with this rule. Parsimony has furthermore been shown to be statistically inconsistent (to arrive at the wrong answer at statistical significance) in a wide range of relatively realistic schemes ${ }^{6-8}$. Parsimony may have its limited set of applications, but for the average phylogeneticist it is not the method of choice.

Further, Stewart states that "[Distance] methods ignore the possibility that apparent overall similarity and true evolutionary relationship are not necessarily the same thing." This statement is incorrect. When properly applied, certain distance methods are powerful methods of phylogenetic inference that make less restrictive assumptions about the evolutionary process than does parsimony. The neighbour-joining method $^{9}$, for example, with properly corrected pairwise distances, is a reliable method of phylogenetic inference even in the absence of a molecular clock ${ }^{8}$.

For nonspecialists, the following may serve as a brief statistical guide for conducting or evaluating phylogenetic sequence analyses. (1) Are the assumptions of the method used met by the mode of evolution of the molecule analysed? Does the method's explicit or implicit stochastic model take account of biased base composition, of different rates of transitions and transversions, or of variations among sites in the rate of evolution, for example? If an analysis was done with parsimony, is its use explicitly justified? (2) Were enough data collected to allow evaluation of multiple internal nodes (those nodes that determine the exact branching pattern of the tree)? (3) Are the conclusions based on significance obtained with a statistical method of phylogenetic inference, such as maximum likelihood, neighbour-joining with properly corrected distances, or invariant analysis? Application of statistical methods of phylogenetic inference avoids many of the pitfalls of parsimony, but has all of its powers.

\section{Arend Sidow}

Department of Molecular Cell Biology,

University of California,

Berkeley, California 94720, USA

STEWART REPLIES - The purpose of my invited Review ${ }^{1}$ was to discuss the powers and pitfails of parsimony analysis in modern molecular evolutionary biology, not to review the numerous methods of phylogenetic inference. For this purpose, it was important to emphasize the key con- cept - which is not always intuitively obvious - that overall similarity measures do not necessarily reflect the true evolutionary relationship of the taxa from which they were made. For the sake of explaining this concept (and realizing that I might anger some advocates of distance methods), I contrasted similarity and relationship in a simple manner, explaining the unique power of parsimony analysis to detect false similarities. Although I did not discuss other methods that attempt to build true phylogenetic trees from molecular data ${ }^{10,11}$, such as maximum likelihood $^{2,3}$, invariant analysis, and distance methods using matrices adjusted for superimposed substitutions ${ }^{4,10,11}$ (Sidow's so-called "statistical" methods), I also did not advocate parsimony to the exclusion of these methods. Indeed, some types of molecular data, such as DNA hybridization and immunological distances, can be analysed only by distance methods ${ }^{10}$. I would again refer the interested reader to the excellent review articles ${ }^{10,12,13}$ mentioned in my review for a more thorough discussion of the various methods of phylogenetic inference.

I also highly recommend a recent article by Huelsenbeck and Hillis ${ }^{14}$, in which 16 distance, invariants and parsimony methods were compared regarding their abilities to find the correct four-taxon tree. (Maximum likelihood was not included in this study because of its computational expense.) This study is noteworthy because the 16 methods were extensively examined under several models of DNA evolution, including ones that conform to and ones that violate the assumptions of the methods. Parsimony methods were generally found to be good at inferring the true phylogeny, so long as the true phylogeny does not suffer from the following conditions: that of having a very short internal lineage separating two clusters, each cluster having one lineage that is quite long relative to its sister lineage. Because few phylogenetically informative events will occur along the short central lineage, homoplastic substitutions on the long lineages tend to group them erroneously during parsimony tree building 6 . This well-characterized phenomenon is termed the "Felsenstein zone" 14 in phylogenetic inference. One important observation that Huelsenbeck and Hillis ${ }^{14}$ make is that all distance methods can fail to find the correct tree in the Felsenstein zone, and many find the wrong tree with certainty. We fully understand why parsimony can fail under these ${ }^{6}$ and some other conditions ${ }^{1,8}$, but we are only beginning to understand exactly when and why distance methods can fail to find correct trees ${ }^{8,14}$.

Another noteworthy point illustrated in ref. 14 is that with highly divergent sequences corrected distances become undefined; distance methods cannot be used in 